\title{
Sharp Integral Inequalities Based on a General Four-Point Quadrature Formula via a Generalization of the Montgomery Identity
}

\author{
J. Pečarić ${ }^{1}$ and M. Ribičić Penava ${ }^{2}$ \\ ${ }^{1}$ Faculty of Textile Technology, University of Zagreb, Pierottijeva 6, 10000 Zagreb, Croatia \\ ${ }^{2}$ Department of Mathematics, University of Osijek, Trg Ljudevita Gaja 6, 31000 Osijek, Croatia
}

Correspondence should be addressed to M. Ribičić Penava, mihaela@mathos.hr

Received 28 March 2012; Accepted 10 July 2012

Academic Editor: Marianna Shubov

Copyright (C) 2012 J. Pečarić and M. Ribičić Penava. This is an open access article distributed under the Creative Commons Attribution License, which permits unrestricted use, distribution, and reproduction in any medium, provided the original work is properly cited.

We consider families of general four-point quadrature formulae using a generalization of the Montgomery identity via Taylor's formula. The results are applied to obtain some sharp inequalities for functions whose derivatives belong to $L_{p}$ spaces. Generalizations of Simpson's 3/8 formula and the Lobatto four-point formula with related inequalities are considered as special cases.

\section{Introduction}

The most elementary quadrature rules in four nodes are Simpson's 3/8 rule based on the following four point formula

$$
\int_{a}^{b} f(t) d t=\frac{b-a}{8}\left[f(a)+3 f\left(\frac{2 a+b}{3}\right)+3 f\left(\frac{a+2 b}{3}\right)+f(b)\right]-\frac{(b-a)^{5}}{6480} f^{(4)}(\xi)
$$

where $\xi \in[a, b]$, and Lobatto rule based on the following four point formula

$$
\int_{-1}^{1} f(t) d t=\frac{1}{6}\left[f(-1)+5 f\left(-\frac{\sqrt{5}}{5}\right)+5 f\left(\frac{\sqrt{5}}{5}\right)+f(1)\right]-\frac{2}{23625} f^{(6)}(\eta)
$$


where $\eta \in[-1,1]$. Formula (1.1) is valid for any function $f$ with a continuous fourth derivative $f^{(4)}$ on $[a, b]$ and formula (1.2) is valid for any function $f$ with a continuous sixth derivative $f^{(6)}$ on $[-1,1]$.

Let $f:[a, b] \rightarrow \mathbb{R}$ be differentiable on $[a, b]$ and $f^{\prime}:[a, b] \rightarrow \mathbb{R}$ integrable on $[a, b]$. Then the Montgomery identity holds (see [1])

$$
f(x)=\frac{1}{b-a} \int_{a}^{b} f(t) d t+\int_{a}^{b} P(x, t) f^{\prime}(t) d t
$$

where the Peano kernel is

$$
P(x, t)= \begin{cases}\frac{t-a}{b-a}, & a \leq t \leq x \\ \frac{t-b}{b-a}, & x<t \leq b .\end{cases}
$$

In [2], Pečarić proved the following weighted Montgomery identity

$$
f(x)=\int_{a}^{b} w(t) f(t) d t+\int_{a}^{b} P_{w}(x, t) f^{\prime}(t) d t
$$

where $w:[a, b] \rightarrow[0, \infty\rangle$ is some probability density function, that is, integrable function, satisfying $\int_{a}^{b} w(t) d t=1$, and $W(t)=\int_{a}^{t} w(x) d x$ for $t \in[a, b], W(t)=0$ for $t<a$ and $W(t)=1$ for $t>b$ and $P_{w}(x, t)$ is the weighted Peano kernel defined by

$$
P_{w}(x, t)= \begin{cases}W(t), & a \leq t \leq x \\ W(t)-1, & x<t \leq b\end{cases}
$$

Now, let us suppose that $I$ is an open interval in $\mathbb{R},[a, b] \subset I, f: I \rightarrow \mathbb{R}$ is such that $f^{(n-1)}$ is absolutely continuous for some $n \geq 2, w:[a, b] \rightarrow[0, \infty\rangle$ is a probability density function. Then the following generalization of the weighted Montgomery identity via Taylor's formula states (given by Aglić Aljinović and Pečarić in [3])

$$
\begin{aligned}
f(x)= & \int_{a}^{b} w(t) f(t) d t-\sum_{i=0}^{n-2} \frac{f^{(i+1)}(x)}{(i+1) !} \int_{a}^{b} w(s)(s-x)^{i+1} d s \\
& +\frac{1}{(n-1) !} \int_{a}^{b} T_{w, n}(x, s) f^{(n)}(s) d s
\end{aligned}
$$

where $x \in[a, b]$ and

$$
T_{w, n}(x, s)= \begin{cases}\int_{a}^{s} w(u)(u-s)^{n-1} d u, & a \leq s \leq x \\ -\int_{s}^{b} w(u)(u-s)^{n-1} d u, & x<s \leq b .\end{cases}
$$


If we take $w(t)=1 /(b-a), t \in[a, b]$, equality (1.7) reduces to

$$
\begin{aligned}
f(x)= & \frac{1}{b-a} \int_{a}^{b} f(t) d t-\sum_{i=0}^{n-2} f^{(i+1)}(x) \frac{(b-x)^{i+2}-(a-x)^{i+2}}{(i+2) !(b-a)} \\
& +\frac{1}{(n-1) !} \int_{a}^{b} T_{n}(x, s) f^{(n)}(s) d s,
\end{aligned}
$$

where $x \in[a, b]$ and

$$
T_{n}(x, s)= \begin{cases}-\frac{(a-s)^{n}}{n(b-a)}, & a \leq s \leq x, \\ -\frac{(b-s)^{n}}{n(b-a)}, & x<s \leq b .\end{cases}
$$

For $n=1$, (1.9) reduces to the Montgomery identity (1.3).

In this paper, we generalize the results from [4]. Namely, we use identities (1.7) and (1.9) to establish for each number $x \in(a,(a+b) / 2]$ a general four-point quadrature formula of the type

$$
\begin{aligned}
\int_{a}^{b} w(t) f(t) d t= & \left(\frac{1}{2}-A(x)\right)[f(a)+f(b)] \\
& +A(x)[f(x)+f(a+b-x)]+R(f, w ; x),
\end{aligned}
$$

where $R(f, w ; x)$ is the remainder and $A:(a,(a+b) / 2] \rightarrow \mathbb{R}$ is a real function. The obtained formula is used to prove a number of inequalities which give error estimates for the general four-point formula for functions whose derivatives are from $L_{p}$-spaces. These inequalities are generally sharp. As special cases of the general non-weighted four-point quadrature formula, we obtain generalizations of the well-known Simpson's 3/8 formula and Lobatto four-point formula with related inequalities.

\section{General Weighted Four-Point Formula}

Let $f:[a, b] \rightarrow \mathbb{R}$ be such that $f^{(n-1)}$ exists on $[a, b]$ for some $n \geq 2$. We introduce the following notation for each $x \in(a,(a+b) / 2]$ :

$$
D(x)=\left(\frac{1}{2}-A(x)\right)[f(a)+f(b)]+A(x)[f(x)+f(a+b-x)]
$$




$$
\begin{aligned}
& t_{w, n}(x)=A(x)\left[\sum_{i=0}^{n-2} \frac{f^{(i+1)}(x)}{(i+1) !} \int_{a}^{b} w(s)(s-x)^{i+1} d s\right. \\
& \left.+\sum_{i=0}^{n-2} \frac{f^{(i+1)}(a+b-x)}{(i+1) !} \int_{a}^{b} w(s)(s-a-b+x)^{i+1} d s\right] \\
& +\left(\frac{1}{2}-A(x)\right)\left[\sum_{i=0}^{n-2} \frac{f^{(i+1)}(a)}{(i+1) !} \int_{a}^{b} w(s)(s-a)^{i+1} d s\right. \\
& \left.+\sum_{i=0}^{n-2} \frac{f^{(i+1)}(b)}{(i+1) !} \int_{a}^{b} w(s)(s-b)^{i+1} d s\right], \\
& \widehat{T}_{w, n}(x, s)=-\left(\frac{1}{2}-A(x)\right)\left[T_{w, n}(a, s)+T_{w, n}(b, s)\right]-A(x)\left[T_{w, n}(x, s)+T_{w, n}(a+b-x, s)\right] \\
& = \begin{cases}-\left(\frac{1}{2}+A(x)\right) \int_{a}^{s} w(u)(u-s)^{n-1} d u & \\
+\left(\frac{1}{2}-A(x)\right) \int_{s}^{b} w(u)(u-s)^{n-1} d u, & a \leq s \leq x, \\
-\frac{1}{2}\left[\int_{a}^{s} w(u)(u-s)^{n-1} d u-\int_{s}^{b} w(u)(u-s)^{n-1} d u\right], & x<s \leq a+b-x, \\
-\left(\frac{1}{2}-A(x)\right) \int_{a}^{s} w(u)(u-s)^{n-1} d u & \\
+\left(\frac{1}{2}+A(x)\right) \int_{s}^{b} w(u)(u-s)^{n-1} d u, & a+b-x<s \leq b .\end{cases}
\end{aligned}
$$

In the next theorem we establish the general weighted four-point formula.

Theorem 2.1. Let I be an open interval in $\mathbb{R},[a, b] \subset I$, and let $w:[a, b] \rightarrow[0, \infty\rangle$ be some probability density function. Let $f: I \rightarrow \mathbb{R}$ be such that $f^{(n-1)}$ is absolutely continuous for some $n \geq 2$. Then for each $x \in(a,(a+b) / 2]$ the following identity holds

$$
\int_{a}^{b} w(t) f(t) d t=D(x)+t_{w, n}(x)+\frac{1}{(n-1) !} \int_{a}^{b} \widehat{T}_{w, n}(x, s) f^{(n)}(s) d s .
$$

Proof. We put $x \equiv a, x \equiv x, x \equiv a+b-x$ and $x \equiv b$ in (1.7) to obtain four new formulae. After multiplying these four formulae by $1 / 2-A(x), A(x), A(x)$ and $1 / 2-A(x)$, respectively, and adding, we get (2.2).

Remark 2.2. Identity (2.2) holds true in the case $n=1$. It can also be obtained by taking $x \equiv a, x \equiv x, x \equiv a+b-x$ and $x \equiv b$ in (1.5), multiplying these four formulae by $1 / 2-$ $A(x), A(x), A(x)$ and $1 / 2-A(x)$, respectively, and adding. In this special case we have

$$
\int_{a}^{b} w(t) f(t) d t=D(x)+\int_{a}^{b} \widehat{T}_{w, 1}(x, s) f^{\prime}(s) d s
$$


where

$$
\begin{aligned}
\widehat{T}_{w, 1}(x, s)= & -\left(\frac{1}{2}-A(x)\right)\left[T_{w, 1}(a, s)+T_{w, 1}(b, s)\right]-A(x)\left[T_{w, 1}(x, s)+T_{w, 1}(a+b-x, s)\right] \\
= & -\left(\frac{1}{2}-A(x)\right)\left[P_{w}(a, s)+P_{w}(b, s)\right]-A(x)\left[P_{w}(x, s)+P_{w}(a+b-x, s)\right] \\
= & \begin{cases}\frac{1}{2}-A(x)-W(s), & a \leq s \leq x, \\
\frac{1}{2}-W(s), & x<s \leq a+b-x, \\
\frac{1}{2}+A(x)-W(s), & a+b-x<s \leq b .\end{cases}
\end{aligned}
$$

Theorem 2.3. Suppose that all assumptions of Theorem 2.1 hold. Additionally, assume that $(p, q)$ is a pair of conjugate exponents, that is, $1 \leq p, q \leq \infty, 1 / p+1 / q=1$, let $f^{(n)} \in L^{p}[a, b]$ for some $n \geq 1$. Then for each $x \in(a,(a+b) / 2]$ we have

$$
\left|\int_{a}^{b} w(t) f(t) d t-D(x)-t_{w, n}(x)\right| \leq \frac{1}{(n-1) !}\left\|\widehat{T}_{w, n}(x, \cdot)\right\|\left\|_{q}\right\| f^{(n)} \|_{p} .
$$

Inequality (2.5) is sharp for $1<p \leq \infty$.

Proof. By applying the Hölder inequality we have

$$
\left|\frac{1}{(n-1) !} \int_{a}^{b} \widehat{T}_{w, n}(x, s) f^{(n)}(s) d s\right| \leq \frac{1}{(n-1) !}\left\|\widehat{T}_{w, n}(x, \cdot)\right\|_{q}\left\|f^{(n)}\right\|_{p} .
$$

By using the above inequality from (2.2) we obtain estimate (2.5). Let us denote $U_{n}^{x}(s)=$ $\widehat{T}_{w, n}(x, s)$. For the proof of sharpness, we will find a function $f$ such that

$$
\left|\int_{a}^{b} U_{n}^{x}(s) f^{(n)}(s) d s\right|=\left\|U_{n}^{x}\right\|_{q}\left\|f^{(n)}\right\|_{p}
$$

For $1<p<\infty$, take $f$ to be such that

$$
f^{(n)}(s)=\operatorname{sign} U_{n}^{x}(s) \cdot\left|U_{n}^{x}(s)\right|^{1 /(p-1)},
$$

where for $p=\infty$ we put

$$
f^{(n)}(s)=\operatorname{sign} U_{n}^{x}(s)
$$

Remark 2.4. Inequality (2.5) for $A(x)=1 / 4$ was proved by Aglić Aljinović et al. in [4]. 


\section{Non-Weighted Four-Point Formula and Applications}

Here we define

$$
\begin{aligned}
\widehat{t}_{n}(x)= & A(x) \sum_{i=0}^{n-2}\left[f^{(i+1)}(x)+(-1)^{i+1} f^{(i+1)}(a+b-x)\right] \frac{(b-x)^{i+2}-(a-x)^{i+2}}{(i+2) !(b-a)} \\
& +\left(\frac{1}{2}-A(x)\right) \sum_{i=0}^{n-2}\left[f^{(i+1)}(a)+(-1)^{i+1} f^{(i+1)}(b)\right] \frac{(b-a)^{i+1}}{(i+2) !}, \\
\widehat{T}_{n}(x, s)= & -n\left\{\left(\frac{1}{2}-A(x)\right)\left[T_{n}(a, s)+T_{n}(b, s)\right]+A(x)\left[T_{n}(x, s)+T_{n}(a+b-x, s)\right]\right\} \\
= & \begin{cases}\left(\frac{1}{2}+A(x)\right) \frac{(a-s)^{n}}{(b-a)}+\left(\frac{1}{2}-A(x)\right) \frac{(b-s)^{n}}{(b-a)}, & a \leq s \leq x, \\
\left(\frac{1}{2}-A(x)\right) \frac{(a-s)^{n}}{(b-a)}+\left(\frac{1}{2}+A(x)\right) \frac{(b-s)^{n}}{(b-a)}, & a+b-x<s \leq b .\end{cases}
\end{aligned}
$$

Theorem 3.1. Let $I$ be an open interval in $\mathbb{R},[a, b] \subset I$, and let $f: I \rightarrow \mathbb{R}$ be such that $f^{(n-1)}$ is absolutely continuous for some $n \geq 1$. Then for each $x \in(a,(a+b) / 2]$ the following identity holds

$$
\frac{1}{b-a} \int_{a}^{b} f(t) d t=D(x)+\widehat{t}_{n}(x)+\frac{1}{n !} \int_{a}^{b} \widehat{T}_{n}(x, s) f^{(n)}(s) d s .
$$

Proof. We take $w(t)=1 /(b-a), t \in[a, b]$ in $(2.2)$.

Theorem 3.2. Suppose that all assumptions of Theorem 3.1 hold. Additionally, assume that $(p, q)$ is a pair of conjugate exponents, that is, $1 \leq p, q \leq \infty, 1 / p+1 / q=1$ and $f^{(n)} \in L^{p}[a, b]$ for some $n \geq 1$. Then for each $x \in(a,(a+b) / 2]$ we have

$$
\left|\frac{1}{b-a} \int_{a}^{b} f(t) d t-D(x)-\widehat{t}_{n}(x)\right| \leq \frac{1}{n !}\left\|\widehat{T}_{n}(x, \cdot)\right\|_{q}\left\|f^{(n)}\right\|_{p} .
$$

Inequality (3.4) is sharp for $1<p \leq \infty$.

Proof. We take $w(t)=1 /(b-a), t \in[a, b]$ in $(2.5)$.

Now, we set

$$
A(x)=\frac{(b-a)^{2}}{12(x-a)(b-x)}, \quad x \in\left(a, \frac{a+b}{2}\right]
$$

This special choice of the function $A$ enables us to consider generalizations of the well-known Simpson's $3 / 8$ formula (1.1) and Lobatto formula (1.2) 
3.1. $x=(2 a+b) / 3$

Suppose that all assumptions of Theorem 3.1 hold. Then the following generalization of Simpson's 3/8 formula reads

$$
\frac{1}{b-a} \int_{a}^{b} f(t) d t=D\left(\frac{2 a+b}{3}\right)+\widehat{t}_{n}\left(\frac{2 a+b}{3}\right)+\frac{1}{n !} \int_{a}^{b} \widehat{T}_{n}\left(\frac{2 a+b}{3}, s\right) f^{(n)}(s) d s,
$$

where

$$
\begin{gathered}
D\left(\frac{2 a+b}{3}\right)=\frac{1}{8}\left(f(a)+3 f\left(\frac{2 a+b}{3}\right)+3 f\left(\frac{a+2 b}{3}\right)+f(b)\right), \\
\widehat{t}_{n}\left(\frac{2 a+b}{3}\right)=\frac{1}{8} \sum_{i=0}^{n-2}\left[f^{(i+1)}\left(\frac{2 a+b}{3}\right)+(-1)^{i+1} f^{(i+1)}\left(\frac{a+2 b}{3}\right)\right] \frac{\left[2^{i+2}+(-1)^{i+1}\right](b-a)^{i+1}}{3^{i+1}(i+2) !} \\
+\frac{1}{8} \sum_{i=0}^{n-2}\left[f^{(i+1)}(a)+(-1)^{i+1} f^{(i+1)}(b)\right] \frac{(b-a)^{i+1}}{(i+2) !}, \\
\widehat{T}_{n}\left(\frac{2 a+b}{3}, s\right)=-\frac{n}{8}\left[T_{n}(a, s)+3 T_{n}\left(\frac{2 a+b}{3}, s\right)+3 T_{n}\left(\frac{a+2 b}{3}, s\right)+T_{n}(b, s)\right] \\
= \begin{cases}\frac{7(a-s)^{n}+(b-s)^{n}}{8(b-a)} & a \leq s \leq \frac{2 a+b}{3}, \\
\frac{(a-s)^{n}+(b-s)^{n}}{2(b-a)}, & \frac{2 a+b}{3}<s \leq \frac{a+2 b}{3}, \\
\frac{(a-s)^{n}+7(b-s)^{n}}{8(b-a)} & \frac{a+2 b}{3}<s \leq b .\end{cases}
\end{gathered}
$$

In the next corollaries we will use the beta function and the incomplete beta function of Euler type defined by

$$
B(x, y)=\int_{0}^{1} t^{x-1}(1-t)^{y-1} d t, \quad B_{r}(x, y)=\int_{0}^{r} t^{x-1}(1-t)^{y-1} d t, \quad x, y>0 .
$$

Corollary 3.3. Suppose that all assumptions of Theorem 3.1 hold. Additionally, assume that $(p, q)$ is a pair of conjugate exponents and $n \in \mathbb{N}$.

(a) If $f^{(n)} \in L^{\infty}[a, b]$, then

$$
\left|\frac{1}{b-a} \int_{a}^{b} f(t) d t-D\left(\frac{2 a+b}{3}\right)\right| \leq \frac{25}{288}(b-a)\left\|f^{\prime}\right\|_{\infty^{\prime}}
$$




$$
\begin{aligned}
\mid \frac{1}{b-a} \int_{a}^{b} f(t) d t & -D\left(\frac{2 a+b}{3}\right)-\widehat{t}_{n}\left(\frac{2 a+b}{3}\right) \mid \\
\leq \frac{1}{(n+1) !}( & \frac{\left[3^{n+1}+3 \cdot 2^{n+1}+3(-1)^{n}\right](b-a)^{n}}{4 \cdot 3^{n+1}} \\
& \left.-\left(\frac{b-a}{2}\right)^{n}\left[\frac{(-1)^{n+1}+1}{2}\right]\right)\left\|f^{(n)}\right\|_{\infty} \quad n \geq 2 .
\end{aligned}
$$

(b) If $f^{(n)} \in L^{2}[a, b]$, then

$$
\begin{aligned}
& \left|\frac{1}{b-a} \int_{a}^{b} f(t) d t-D\left(\frac{2 a+b}{3}\right)-\widehat{t}_{n}\left(\frac{2 a+b}{3}\right)\right| \\
& \leq \frac{1}{n !}\left(\frac{\left[3^{2 n}+5 \cdot 2^{2 n+1}+11\right](b-a)^{2 n-1}}{32 \cdot 3^{2 n}(2 n+1)}+\frac{(-1)^{n}(b-a)^{2 n-1}}{32}\right. \\
& \left.\quad \times\left[7 B(n+1, n+1)+9 B_{2 / 3}(n+1, n+1)-9 B_{1 / 3}(n+1, n+1)\right]\right)^{1 / 2}\left\|f^{(n)}\right\|_{2} .
\end{aligned}
$$

(c) If $f^{(n)} \in L^{1}[a, b]$, then

$$
\left|\frac{1}{b-a} \int_{a}^{b} f(t) d t-D\left(\frac{2 a+b}{3}\right)-\widehat{t}_{n}\left(\frac{2 a+b}{3}\right)\right| \leq \frac{1}{n !} K_{n}\left(\frac{2 a+b}{3}\right)\left\|f^{(n)}\right\|_{1^{\prime}}
$$

where $K_{1}((2 a+b) / 3)=5 / 24, K_{2}((2 a+b) / 3)=(5 / 18)(b-a), K_{3}((2 a+b) / 3)=(7 / 54)(b-a)^{2}$ and $K_{n}((2 a+b) / 3)=(1 / 8)(b-a)^{n-1}$, for $n \geq 4$.

The first and the second inequality are sharp.

Proof. We apply (3.4) with $x=(2 a+b) / 3$ and $p=\infty$

$$
\begin{aligned}
\int_{a}^{b}\left|\widehat{T}_{n}\left(\frac{2 a+b}{3}, s\right)\right| d s= & \int_{a}^{(2 a+b) / 3}\left|\frac{7(a-s)^{n}+(b-s)^{n}}{8(b-a)}\right| d s \\
& +\int_{(2 a+b) / 3}^{(a+2 b) / 3}\left|\frac{(a-s)^{n}+(b-s)^{n}}{2(b-a)}\right| d s+\int_{(a+2 b) / 3}^{b}\left|\frac{(a-s)^{n}+7(b-s)^{n}}{8(b-a)}\right| d s \\
= & 2 \frac{\left[3^{n+1}-2^{n+1}+7 \cdot(-1)^{n}\right](b-a)^{n}}{8 \cdot 3^{n+1}(n+1)}
\end{aligned}
$$


International Journal of Mathematics and Mathematical Sciences

$$
\begin{aligned}
& +\frac{\left(2^{n+1}+(-1)^{n+1}\right)(b-a)^{n}}{3^{n+1}(n+1)}-\frac{\left(1+(-1)^{n+1}\right)(b-a)^{n}}{2^{n+1}(n+1)} \\
= & \frac{\left[3^{n+1}+3 \cdot 2^{n+1}+3(-1)^{n}\right](b-a)^{n}}{4 \cdot 3^{n+1}(n+1)}-\left(\frac{b-a}{2}\right)^{n}\left[\frac{(-1)^{n+1}+1}{2(n+1)}\right],
\end{aligned}
$$

for $n \geq 2$ and

$$
\int_{a}^{b}\left|\widehat{T}_{1}\left(\frac{2 a+b}{3}, s\right)\right| d s=\frac{25}{288}(b-a) .
$$

To obtain the second inequality we take $p=2$

$$
\begin{aligned}
\int_{a}^{b}\left|\widehat{T}_{n}\left(\frac{2 a+b}{3}, s\right)\right|^{2} d s= & \int_{a}^{(2 a+b) / 3}\left|\frac{7(a-s)^{n}+(b-s)^{n}}{8(b-a)}\right|^{2} d s \\
& +\int_{(2 a+b) / 3}^{(a+2 b) / 3}\left|\frac{(a-s)^{n}+(b-s)^{n}}{2(b-a)}\right|^{2} d s+\int_{(a+2 b) / 3}^{b}\left|\frac{(a-s)^{n}+7(b-s)^{n}}{8(b-a)}\right|^{2} d s \\
= & \frac{\left[3^{2 n}+5 \cdot 2^{2 n+1}+11\right](b-a)^{2 n-1}}{32 \cdot 3^{2 n}(2 n+1)}+\frac{(-1)^{n}(b-a)^{2 n-1}}{32} \\
& \times\left[7 B(n+1, n+1)+9 B_{2 / 3}(n+1, n+1)-9 B_{1 / 3}(n+1, n+1)\right] .
\end{aligned}
$$

If $p=1$, we have

$$
\begin{array}{r}
\sup _{s \in[a, b]}\left|\widehat{T}_{n}\left(\frac{2 a+b}{3}, s\right)\right|=\max \left\{\sup _{s \in[a,(2 a+b) / 3]}\left|\frac{7(a-s)^{n}+(b-s)^{n}}{8(b-a)}\right|,\right. \\
\sup _{s \in[(2 a+b) / 3,(a+2 b) / 3]}\left|\frac{(a-s)^{n}+(b-s)^{n}}{2(b-a)}\right| \\
\left.\sup _{s \in[(a+2 b) / 3, b]}\left|\frac{(a-s)^{n}+7(b-s)^{n}}{8(b-a)}\right|\right\} .
\end{array}
$$

By an elementary calculation we get

$$
\sup _{s \in[a,(2 a+b) / 3]}\left|\frac{7(a-s)+(b-s)}{8(b-a)}\right|=\sup _{s \in[(a+2 b) / 3, b]}\left|\frac{(a-s)+7(b-s)}{8(b-a)}\right|=\frac{5}{24}(b-a),
$$




$$
\begin{aligned}
& \sup _{s \in[a,(2 a+b) / 3]}\left|\frac{7(a-s)^{2}+(b-s)^{2}}{8(b-a)}\right|=\sup _{s \in[(a+2 b) / 3, b]}\left|\frac{(a-s)^{2}+7(b-s)^{2}}{8(b-a)}\right|=\frac{11}{72}(b-a), \\
& \sup _{s \in[a,(2 a+b) / 3]}\left|\frac{7(a-s)^{n}+(b-s)^{n}}{8(b-a)}\right|=\sup _{s \in[(a+2 b) / 3, b]}\left|\frac{(a-s)^{n}+7(b-s)^{n}}{8(b-a)}\right|=\frac{(b-a)^{n-1}}{8},
\end{aligned}
$$

for $n \geq 3$. The function $y:[a, b] \rightarrow \mathbb{R}, y(x)=(a-x)^{n}+(b-x)^{n}$, is decreasing on $\langle a,(a+b) / 2\rangle$ and increasing on $\langle(a+b) / 2, b\rangle$ if $n$ is even, and decreasing on $\langle a, b\rangle$ if $n$ is odd. Thus

$$
\sup _{s \in[(2 a+b) / 3,(a+2 b) / 3]}\left|\frac{(a-s)^{n}+(b-s)^{n}}{2(b-a)}\right|=\frac{\left((-1)^{n}+2^{n}\right)(b-a)^{n-1}}{2 \cdot 3^{n}} .
$$

Finally,

$$
\sup _{s \in[a, b]}\left|\widehat{T}_{1}\left(\frac{2 a+b}{3}, s\right)\right|=\frac{5}{24}
$$

and for $n \geq 2$

$$
\sup _{s \in[a, b]}\left|\widehat{T}_{n}\left(\frac{2 a+b}{3}, s\right)\right|=(b-a)^{n-1} \max \left\{\frac{1}{8}, \frac{2^{n}+(-1)^{n}}{2 \cdot 3^{n}}\right\} .
$$

3.2. $[a, b]=[-1,1], x=-\sqrt{5} / 5$

Suppose that all assumptions of Theorem 3.1 hold. Then the following generalization of Lobatto formula reads

$$
\frac{1}{2} \int_{-1}^{1} f(t) d t=D\left(-\frac{\sqrt{5}}{5}\right)+\widehat{t}_{n}\left(-\frac{\sqrt{5}}{5}\right)+\frac{1}{n !} \int_{-1}^{1} \widehat{T}_{n}\left(-\frac{\sqrt{5}}{5}, s\right) f^{(n)}(s) d s
$$

where

$$
\begin{aligned}
D\left(-\frac{\sqrt{5}}{5}\right)= & \frac{1}{12}\left(f(-1)+5 f\left(-\frac{\sqrt{5}}{5}\right)+5 f\left(\frac{\sqrt{5}}{5}\right)+f(1)\right) \\
\widehat{t}_{n}\left(-\frac{\sqrt{5}}{5}\right)= & \frac{5}{12} \sum_{i=0}^{n-2}\left[f^{(i+1)}\left(-\frac{\sqrt{5}}{5}\right)+(-1)^{i+1} f^{(i+1)}\left(\frac{\sqrt{5}}{5}\right)\right] \\
& \times \frac{(5+\sqrt{5})^{i+2}+(-1)^{i+1}(5-\sqrt{5})^{i+2}}{2 \cdot 5^{i+2}(i+2) !} \\
& +\frac{1}{12} \sum_{i=0}^{n-2}\left[f^{(i+1)}(-1)+(-1)^{i+1} f^{(i+1)}(1)\right] \frac{2^{i+1}}{(i+2) !}
\end{aligned}
$$


International Journal of Mathematics and Mathematical Sciences

$$
\begin{aligned}
\widehat{T}_{n}\left(-\frac{\sqrt{5}}{5}, s\right)= & -\frac{n}{12}\left[T_{n}(-1, s)+5 T_{n}\left(-\frac{\sqrt{5}}{5}, s\right)+5 T_{n}\left(\frac{\sqrt{5}}{5}, s\right)+T_{n}(1, s)\right] \\
& = \begin{cases}\frac{11(-1-s)^{n}+(1-s)^{n}}{24} & -1 \leq s \leq-\frac{\sqrt{5}}{5} \\
\frac{(-1-s)^{n}+(1-s)^{n}}{4} & -\frac{\sqrt{5}}{5}<s \leq \frac{\sqrt{5}}{5} \\
\frac{(-1-s)^{n}+11(1-s)^{n}}{24} & \frac{\sqrt{5}}{5}<s \leq 1 .\end{cases}
\end{aligned}
$$

Corollary 3.4. Suppose that all assumptions of Theorem 3.1 hold. Additionally, assume that $(p, q)$ is a pair of conjugate exponents and $n \in \mathbb{N}$.

(a) if $f^{(n)} \in L^{\infty}[-1,1]$, then

$$
\begin{gathered}
\left|\frac{1}{2} \int_{-1}^{1} f(t) d t-D\left(-\frac{\sqrt{5}}{5}\right)\right| \leq\left(\frac{101}{180}-\frac{\sqrt{5}}{6}\right)\left\|f^{\prime}\right\|_{\infty^{\prime}} \\
\left|\frac{1}{2} \int_{-1}^{1} f(t) d t-D\left(-\frac{\sqrt{5}}{5}\right)-\widehat{t}_{n}\left(-\frac{\sqrt{5}}{5}\right)\right| \\
\leq \frac{1}{(n+1) !}\left(\frac{2^{n+1} \cdot 5^{n}+(5+\sqrt{5})^{n+1}-(-5+\sqrt{5})^{n+1}}{12 \cdot 5^{n}}\right. \\
\left.-\frac{1+(-1)^{n+1}}{2}\right)\left\|f^{(n)}\right\|_{\infty}, \quad n \geq 2 .
\end{gathered}
$$

(b) if $f^{(n)} \in L^{2}[-1,1]$, then

$$
\begin{aligned}
& \mid \frac{1}{2} \int_{-1}^{1} f(t) d t-D\left(-\frac{\sqrt{5}}{5}\right)-\widehat{t}_{n}\left(-\frac{\sqrt{5}}{5}\right) \mid \\
& \leq \frac{1}{n !} \cdot \frac{2^{n-2}}{3}\left(\frac{35(5+\sqrt{5})^{2 n+1}+85(5-\sqrt{5})^{2 n+1}+10^{2 n+1}}{10^{2 n+1}(2 n+1)}\right. \\
&+(-1)^{n}\left[11 B(n+1, n+1)+25 B_{(5+\sqrt{ } 5) / 10}(n+1, n+1)\right. \\
&\left.\left.-25 B_{(5-\sqrt{5}) / 10}(n+1, n+1)\right]\right)^{1 / 2}\left\|f^{(n)}\right\|_{2} .
\end{aligned}
$$


(c) if $f^{(n)} \in L^{1}[-1,1]$, then

$$
\left|\frac{1}{2} \int_{-1}^{1} f(t) d t-D\left(-\frac{\sqrt{5}}{5}\right)-\widehat{t}_{n}\left(-\frac{\sqrt{5}}{5}\right)\right| \leq \frac{1}{n !} K_{n}\left(-\frac{\sqrt{5}}{5}\right)\left\|f^{(n)}\right\|_{1^{\prime}}
$$

where $K_{1}(-\sqrt{5} / 5)=1 /(2 \sqrt{5}), K_{2}(-\sqrt{5} / 5)=3 / 5, K_{3}(-\sqrt{5} / 5)=8 /(5 \sqrt{5}), K_{4}(-\sqrt{5} / 5)=28 / 25$, $K_{5}(-\sqrt{5} / 5)=88 /(25 \sqrt{5}), K_{n}(-\sqrt{5} / 5)=2^{n-3} / 3$, for $n \geq 6$.

The first and the second inequality are sharp.

Proof. Applying (3.4) with $[a, b]=[-1,1], x=-\sqrt{5} / 5$ and $p=\infty, p=2, p=1$ and carrying out the same analysis as in Corollay 3.3 we obtain the above inequalities.

\section{References}

[1] D. S. Mitrinović, J. E. Pečarić, and A. M. Fink, Inequalities Involving Functions and Their Integrals and Derivatives, vol. 53 of Mathematics and its Applications (East European Series), Kluwer Academic Publishers, Dordrecht, The Netherlands, 1991.

[2] J. E. Pečarić, "On the Čebyšev inequality," Buletinul Ştiinţific al Universităţii Politehnica din Timişoara. Seria Matematică-Fizică, vol. 25, no. 39, pp. 10-11, 1980.

[3] A. Aglić Aljinović and J. Pečarić, "On some Ostrowski type inequalities via Montgomery identity and Taylor's formula," Tamkang Journal of Mathematics, vol. 36, no. 3, pp. 199-218, 2005.

[4] A. Aglić Aljinović, J. Pečarić, and M. Ribičić Penava, "Sharp integral inequalities based on general two-point formulae via an extension of Montgomery's identity," The ANZIAM Journal, vol. 51, no. 1, pp. 67-101, 2009. 


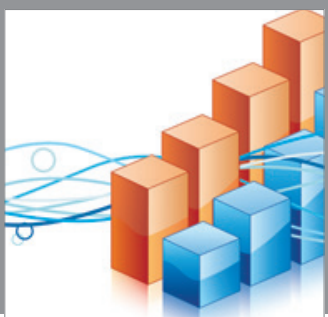

Advances in

Operations Research

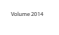

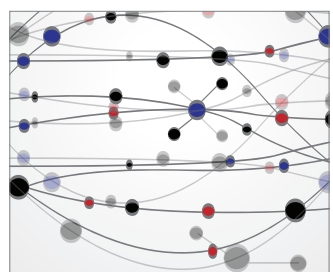

\section{The Scientific} World Journal
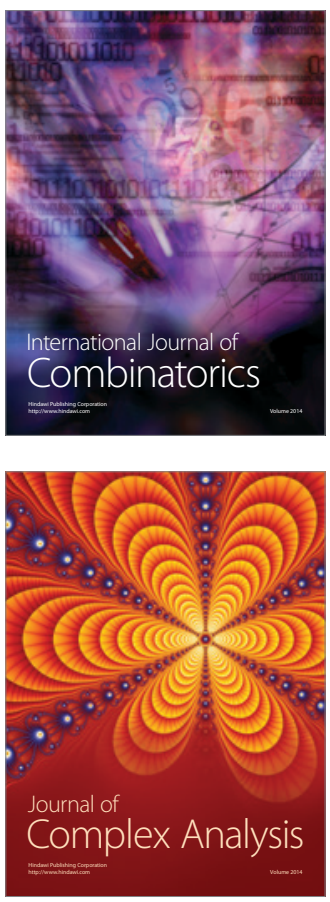

International Journal of

Mathematics and

Mathematical

Sciences
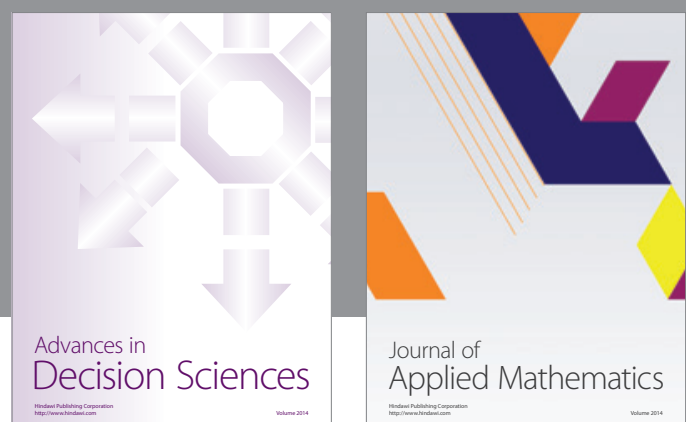

Journal of

Applied Mathematics
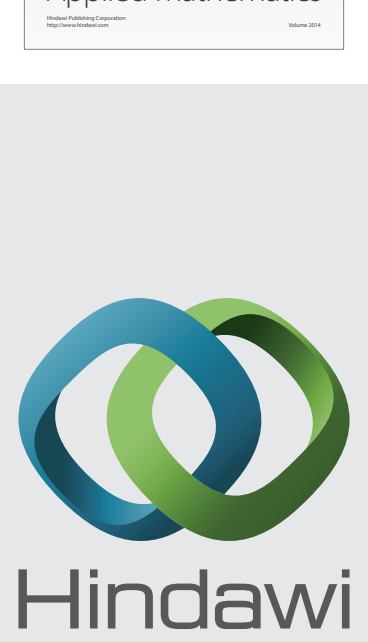

Submit your manuscripts at http://www.hindawi.com
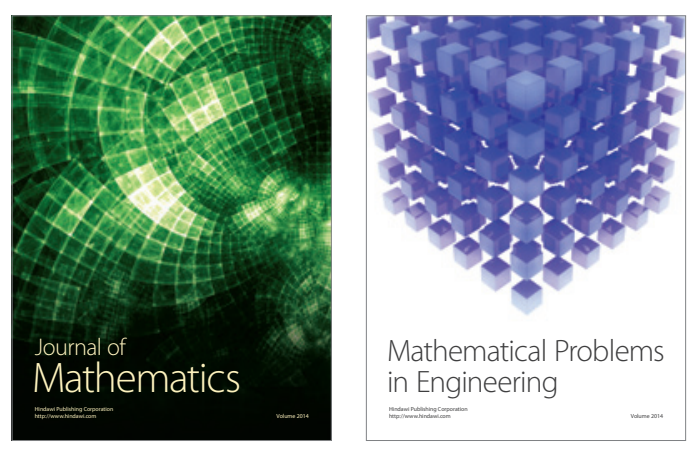

Mathematical Problems in Engineering
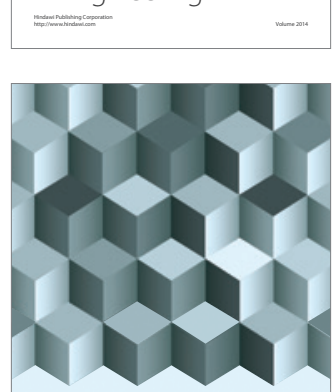

Journal of

Function Spaces
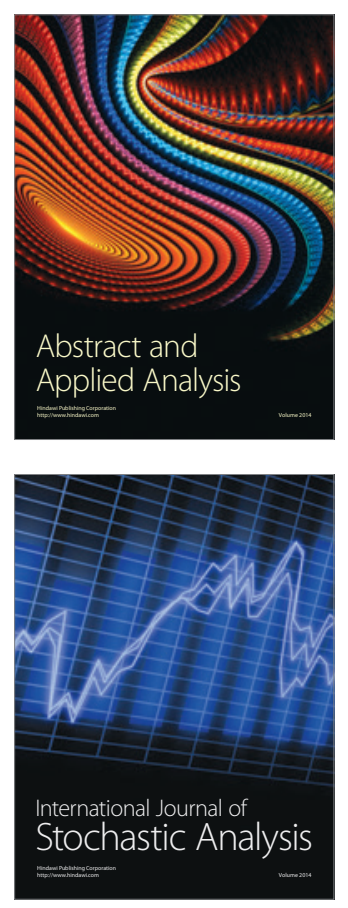

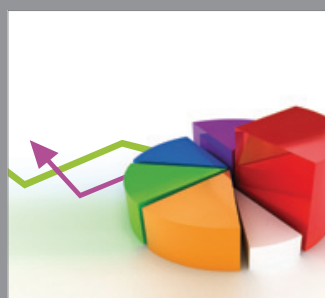

ournal of

Probability and Statistics

Promensencen
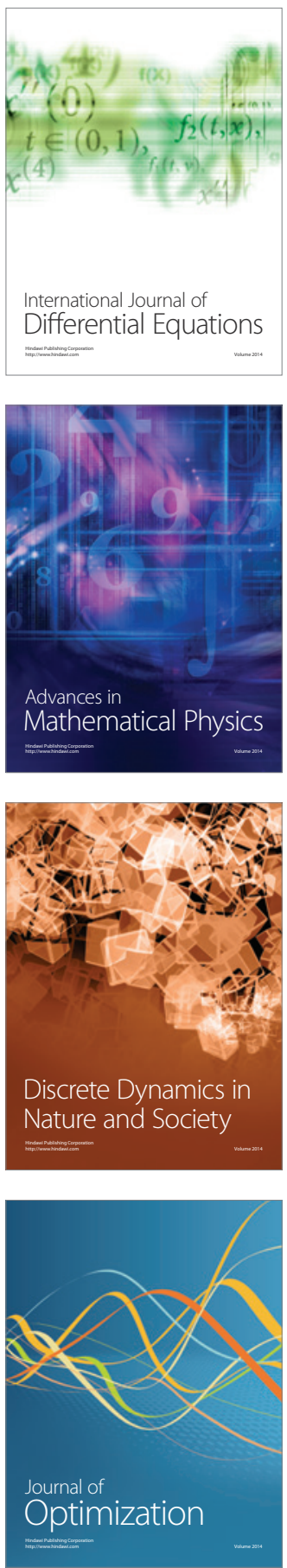